\title{
PPINOT Tool Suite: A Performance Management Solution for Process-Oriented Organisations ${ }^{\star}$
}

\author{
Adela del-Río-Ortega, Cristina Cabanillas, Manuel Resinas, and \\ Antonio Ruiz-Cortés \\ Universidad de Sevilla, Spain \\ \{adeladelrio, resinas, aruiz\}@us.es \\ cristina.cabanillas@wu.ac.at
}

\begin{abstract}
A key aspect in any process-oriented organisation is the measurement of process performance for the achievement of its strategic and operational goals. Process Performance Indicators (PPIs) are a key asset to carry out this evaluation, and, therefore, the management of these PPIs throughout the whole BP lifecycle is crucial. In this demo we present PPINOT Tool Suite, a set of tools aimed at facilitating and automating the PPI management. The support includes their definition using either a graphical or a template-based textual notation, their automated analysis at design-time, and their automated computation based on the instrumentation of a Business Process Management System.
\end{abstract}

\section{Defining and Analysing PPIs with PPINOT}

Performance requirements on business processes (BPs) are usually specified in terms of Process Performance Indicators (PPIs), which are quantifiable metrics that can be measured directly by data that is generated within the process flow and are aimed at evaluating the efficiency and effectiveness of business process.

The management of those PPIs is, thus, an important part of the BP lifecycle that includes at least the definition of PPIs, their analysis to find relationships between them, the instrumentation of the information systems that support the BPs in order to take the measures that are necessary to calculate the PPIs, the actual calculation of the PPIs during process execution, and the monitoring of the PPIs fulfillment.

The PPINOT Metamodel [1] provides a foundation on which an automated support for these activities can be built. It identifies the concepts that are necessary for defining Process Performance Indicators (PPIs) such as the different

\footnotetext{
* This work was partially supported by the European Commission (FEDER), the Spanish and the Andalusian R\&D\&I programmes (grants TIN2009-07366 (SETI), TIN2012-32273 (TAPAS), TIC-5906 (THEOS)). The authors thank the PPINOT development team at the ISA group, for their development work; concretely Ana Belén Sánchez and Edelia García.
} 
types of measures that can be used to compute the PPI value. It was defined to address the challenge of providing PPI definitions that are unambiguous and complete, traceable to the business process elements used in their definition, independent of the language used to model business processes (BP) and amenable to automated analysis.

Two notations have been developed on top of the PPINOT Metamodel, namely a graphical notation and a template-based textual notation [2]. The former extends BPMN to allow the graphical definition of PPIs together with their corresponding BPs. The latter provides a template for PPIs and a set of linguistic patterns the user must follow to make the PPI definition easier [2]. Figure 1 shows an example of a PPI defined using PPINOT. The left hand side corresponds to a template-based definition, the right hand side depicts the corresponding XML serialisation of the PPINOT Metamodel.

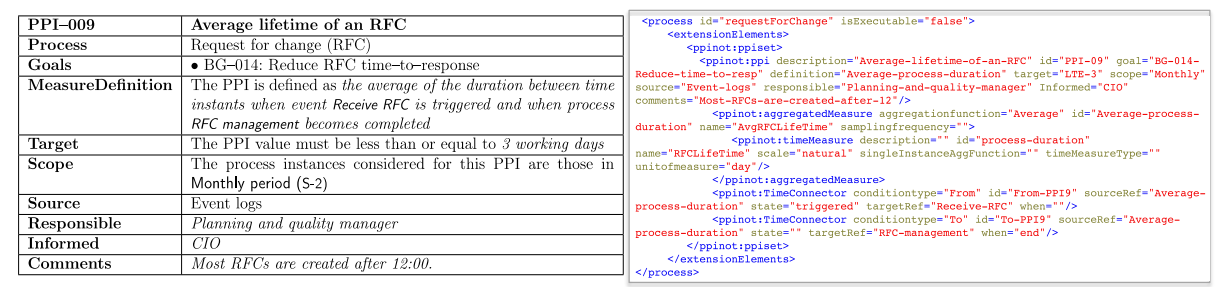

Fig. 1. An example of a PPI defined with PPINOT

The PPINOT Metamodel is also provided with formal semantics based on Description Logics (DL). This enables leveraging reasoning operations implemented by off-the-shelf DL reasoners to automatically extract information from the relationships between PPIs and BP elements. This information can be used to assist process analysts in the definition and instrumentation of PPIs [1].

In this paper, we give an overview of the PPINOT Tool Suite, which is a set of tools based on the PPINOT Metamodel that automate or facilitate some of the aforementioned PPI management activities.

\section{PPINOT Tool Suite Overview}

Figure 2 overviews the tools that compose the PPINOT Tool Suite. Rectangles depict tools, their inputs and outputs are represented by documents linked with dashed arrows, and the possible interconnections between tools is done by using solid arrows. The tools can be used separately or sequentially, depending on the user purposes. In the following we describe a possible way to use it.

Design. The PPINOT Tool Suite offers two different ways to define PPIs. On the one hand, we can define them graphically together with the BPMN representation of the BP using the PPINOT Graphical Editor, which is a web editor 


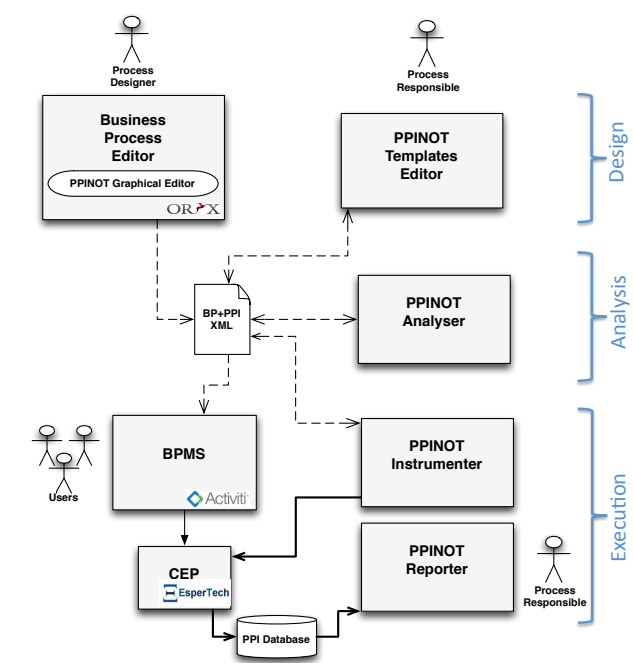

Fig. 2. PPINOT Tool Suite Overview

that has been implemented as an extension of the Oryx platform [3]. On the other hand, we can use the PPINOT Templates Editor to define PPIs using the template-based textual notation. The tool guides the user by providing linguistic patterns according to the selection performed in the different fields. In both cases, an XML document with the PPI information together with the BP model is obtained as output.

Analysis. After defining the PPIs, the PPINOT Analyser can be used. It uses the DL formalisation of PPINOT metamodel to implement the analysis operations that obtain information about the way PPIs and BP elements influence each other. Concretely, two kinds of analysis operations are currently supported: (I) BPElements involved, which allows answering the question Given a PPI, Which are the process model's elements involved?. This information is useful for instance when a PPI must be replaced with others (maybe because it is very costly to obtain its value) and it is necessary to assure that every element of the BP that was measured before is measured in the new case; and (II) PPIs associated to BPElement, which allows answering the question Given a BPElement, Which are the PPIs associated or applied to it? This information can assist during the evolution of BPs (e.g., an activity is deleted) to identify which PPIs will be affected and should be updated.

Execution. The last set of tools that can be used are those focused on the execution of the BP. Before execution, the PPINOT Instrumenter configures Activiti (an open source BPMS ${ }^{1}$ ) to send events to a Complex Event Processor (CEP) and also configures the CEP to compute the values of the defined PPIs

\footnotetext{
${ }^{1}$ http://activiti.org.
} 
from the events generated by Activiti during BP execution. The computed values of the PPIs are stored in a PPI Database. Finally, the PPINOT Reporter can be used to present the user these values ${ }^{2}$.

Further information about PPINOT Tool Suite, the description of the tools and user instructions can be found at http://www.isa.us.es/ppinot.

\section{Significance and Features}

PPINOT Tool Suite has been developed from research results, and validated and extended thanks to the feedback obtained from several real scenarios, both from organisations and academia. To the best of our knowledge, there not exists any similar tool for the definition and design-time analysis of PPIs. Concretely, we can highlight the following features, from which the last four provide the novelty to our proposal:

BPMN 2.0 compliant. PPIs can be defined over BP diagrams (BPDs) previously modelled using the de facto standard BPMN 2.0.

PPI values computation Taking as starting point any of the aforementioned PPI definitions, PPINOT also provides the possibility to extract the information required to calculate PPI values from Activiti, an open source BP management platform, and to create reports with these values.

Graphical definition of PPIs. PPINOT Tool Suite supports the graphical definition of PPIs using a graph-based graphical notation that is easily understandable by non-technical users, at the same time that it is supported by a metamodel that assures the precise and complete definition of PPIs.

Template-based definition of PPIs. PPIs can be defined by fulfilling templates written in structured natural language, where the user only has to properly introduce the missing information, assisted by linguistic patterns.

PPI definition mapping Graphical definition of PPIs can be mapped to their corresponding templates in natural language.

Automated analysis of PPIs. The aforementioned analysis operations can be automatically performed on PPI definitions.

\section{References}

1. del Río-Ortega, A., Resinas, M., Cabanillas, C., Ruiz-Cortés, A.: On the Definition and Design-time Analysis of Process Performance Indicators. Information Systems 38(4) (2012) 470-490

2. del Río-Ortega, A., Resinas, M., Durán, A., Ruiz-Cortés, A.: Defining process performance indicators by using templates and patterns. In: Proc. of the 10th International Conference on Business Process Management (BPM). (2012) 223-228

3. Decker, G., Overdick, H., Weske, M.: Oryx - an open modeling platform for the bpm community. In: Proc. of the 6th International Conference on Business Process Management (BPM). (2008) 382-385

${ }^{2}$ In its current version this tool provides a simple list of values. An extension to provide a proper report is planned. 